\title{
Accelerated Immune Senescence and Biological Aging of T-cell Subsets Among ART-naive HIV- infected Men Who Have Sex With Men in Beijing, China: a Case-control Study
}

\section{Li Li}

Capital Medical University Affiliated Beijing Ditan Hospital https://orcid.org/0000-0002-6666-3830 Cheng-jie Ma

Capital Medical University Affiliated Beijing Ditan Hospital Ling-hang Wang

Capital Medical University Affiliated Beijing Ditan Hospital

\section{Feng-ting Yu}

Capital Medical University Affiliated Beijing Ditan Hospital

\section{Si-yuan Yang}

Capital Medical University Affiliated Beijing Ditan Hospital

\section{Shu-jing Song}

Capital Medical University Affiliated Beijing Ditan Hospital

\section{Yun-xia Tang}

Capital Medical University Affiliated Beijing Ditan Hospital

Xing-wang Li ( $\nabla$ ditanlxw@163.com )

Capital Medical University Affiliated Beijing Ditan Hospital https://orcid.org/0000-0002-2464-9002

\section{Research Article}

Keywords: HIV, ART naive, T-cell, premature aging, immune senescence, immune activation, telomere length

Posted Date: June 8th, 2021

DOl: https://doi.org/10.21203/rs.3.rs-585387/v1

License: (9) (1) This work is licensed under a Creative Commons Attribution 4.0 International License. Read Full License 


\section{Abstract}

Background: The average lifespan of HIV-infected subjects remains shorter compared to uninfected individuals. Accelerated senescence may be responsible for this difference despite effective antiretroviral therapy (ART) with successful viral suppression. The present study aimed to assess the impact of HIV on immune and biological senescence of ART-naive HIV-infected men who have sex with men (MSM) in Beijing, China.

Methods: A cross-sectional study was conducted that compared MSM HIV-infected patients and agematched MSM HIV negative controls. Within the CD4 and CD8 population, the percentages of naive(TN), central memory(TCM), effector memory(TEM) and terminally differentiated memory(TemRA) subsets were studied and markers of senescence and activation in these cells were measured by multiparameter flow cytometry. Naive (CD45RA $\left.{ }^{+}\right)$and memory $\left(C D 45 R 0^{+}\right)$CD8 T cells were purified by MACS technology. Telomere length was quantified by real-time PCR.

Results: Within the CD8 T cell subsets, TN, TCM,TEM and TemRA cells showed more activation (HLA$\left.\mathrm{DR}^{+}\right)$and replicative senescence $\left(\mathrm{CD} 28^{-} \mathrm{CD} 57^{+}\right)$phenotypes in ART-naive MSM. However, in the CD4 T cell subsets, expect for TN, the percentage of senescent cells did not differ between ART-naive MSM and uninfected controls, but activated cells were upregulated in the former. The telomere length of naive and memory CD8 T cells was significantly shorter in ART-naive MSM than that of uninfected controls.

Conclusion: Our results indicate that HIV-infected ART-naive MSM exhibit accelerated immune senescence with premature biological aging, which particularly affects the CD8 T-cell subsets. This highlights the strong impact of HIV on aging process of T-cell despite patient's young age at infection and supports the importance of early control of HIV replication.

\section{Background}

Treatment of HIV-infected patients with combination antiretroviral therapy (ART) has led to a significant reduction in both morbidity and mortality. HIV infection has transitioned from an acute, terminal illness to a chronic but manageable condition [1]. While this is undoubtedly a major success, several studies have demonstrated that HIV-infected individuals are at an increased risk of age-related non-AIDS morbidity and mortality compared with uninfected persons [2]. These observations have led to the proposal that HIVinfected individuals suffer from accelerated or premature aging [3]. However, the pathogenic mechanisms underlying this increased aging process remain poorly understood.

Growing evidence suggests that immune exhaustion and senescence caused by HIV infection is similar to that caused by age in uninfected elderly subjects [4-6]. During nature aging, a reduction in $T$ cell renewal occurs together with a progressive enrichment of terminally differentiated $T$ cells. It is thought that these changes are the consequence of immune activation and inflammation, which translates into a general decline of the immune system, which gradually leads to immunosenescence (aging of the immune system) [7]. Similarly, high levels of systemic immune activation and inflammation due to HIV 
infection promote the accelerated replicative senescence of T cells [8], which leads to an imbalance of T cell phenotype $[9,10]$. This results in the differentiation and accumulation of nonfunctional senescent $T$ cells [5]. On the other hand, a direct consequence of cellular replication is the shortening of their telomeres until they reach a critical length[11-13], triggering premature senescence of cells and the development of those age-related diseases[14]. It has been also advanced that HIV-infected individuals have overall shorter telomeres than uninfected controls[15-18] which occurs early after infection[19].

Although some reports have characterized distinct T cell subsets in HIV-infected subjects and compared their distribution with those in healthy subjects[20, 21], few data are available regarding the activation/senescent profile in relation to the immunosenescence features of distinct $T$ cell subsets in HIV-infected MSM. In addition, telomere length in previous studies was usually measured in total PBMC or in total T cell subsets (i.e. CD4 or CD8), no reports have provided a comprehensive assessment of distinct $T$ cell subsets according to the stage of differentiation. In the current study, we provided a detailed characterization of biological aging in relation to immune senescence and activation markers of the distinct T cell subsets in a cohort of ART-naive HIV-infected MSM populations without the confounding factor of ART with a well matched HIV negative MSM group. Furthermore, we aimed to assess the contribution of HIV to premature aging in ART-naive patients.

\section{Methods}

\section{Study design and participants}

This cross-sectional study was conducted at the acquired immune deficiency syndrome (AIDS) clinical care centre of Beijing Ditan Hospital from March 2018 to June 2019. To be eligible to participate in the study, MSM had to be male, aged 20-40 years, have had sexual contact with men in the previous 12 months and were willing to provide written informed consent. All participants had a confirmed serological diagnosis of HIV and about to commence antiretroviral therapy (ART-naive). Using frequency-matching by sex and 5-year age categories, healthy volunteers were recruited as control group (HCs) from participants confirmed to be HIV-seronegative attending an HIV prevention trial.

The exclusion criteria consisted of acute or chronic HBV or HCV infection, immune inflammatory diseases, advanced stages of severe chronic diseases, such as atherosclerotic disease, congestive heart failure, poorly controlled diabetes mellitus, renal or hepatic disease, chronic obstructive pulmonary disease, as well as individuals under immunosuppressive therapy. This study was approved by the Ethics Committee of the Beijing Ditan Hospital. Written informed consent was obtained from all participants.

\section{Laboratory measurements}

Fasting venous blood samples were collected. CD4 and CD8 T cell counts (cells/ul) were determined in all subjects, while HIV-1 viral load (HIV-RNA, copies/ml) were determined only in HIV-infected subjects. Absolute CD 4 and CD 8 T cell counts were determined using the flow cytometer-500 (Beckman Coulter Inc $\mathrm{CA})$, according to the manufacturer instructions. Plasma HIV-1 RNA levels were determined using the 
COBAS Taqman HIV-1 test (Roche, Branchburg, New Jersey, USA) according to the manufacturer's instructions. The detection limit was 40 HIV RNA copies/mL.

\section{Flow Cytometry analysis}

All analyses were performed using fresh peripheral blood mononuclear cell (PBMC) samples, isolated by density centrifugation using Ficoll-Hypaque (Amersham Biosciences, Amersham, Buckinghamshire, United Kingdom) from $20 \mathrm{ml}$ EDTA venous blood. The following monoclonal antibodies were used for T cell immunophenotyping: CD4-APC-CY7, CD8-FITC, CD28-APC, CD45RA-PE-CY7, CCR7-PERCP-CY5.5, CD27-AmCyan, HLA-DR-Pacific Blue, and CD57-PE(BD Biosciences, San Jose, CA). Combinations of CD45RA and CD27 are commonly employed to define four different $T$ cell subsets[22]: 1) CD45RA ${ }^{+} C D 27^{+}$ [naive T cells (TN)]; 2) CD45RA-CD27 ${ }^{+}$[central memory T cells (TCM)]; 3) CD45RA ${ }^{-C D} 27$-[effector memory T cells (TEM)]; and 4) CD45RA ${ }^{+}$CD27-[terminally differentiated memory T cells (TemRA)]. Immune activation on CD4 and CD8 cell subsets was characterized by by HLA-DR expression[23]. Senescence on CD4 and CD8 cell subsets was assessed by $\mathrm{CD} 57$ and CD28 expression $\left(\mathrm{CD} 57^{+} \mathrm{CD} 28^{-}\right.$measuring senescent T lymphocytes)[22]. Fluorescence was measured with a FACS Canto II (BD Biosciences, Breda, the Netherlands). A total of 100,000 events were collected in the lymphocyte gate using morphological parameters (forward and side-scatter). The raw data were analysed using a fluorescence activated cell sorter (FACS) Diva version 5 (BD Biosciences, Heidelberg, Germany) and FlowJo software (TreeStar Inc., Ashland, OR, USA).

\section{Isolation of naive and memory CD8 T cells}

CD8 T cells were obtained by MACS selection from fresh PBMCs using negative selection MACS-kits according to manufacturer's instructions (CD8 T cell Isolation Kit; Miltenyi). In a second step, CD45RA ${ }^{+}$ and $\mathrm{CD} 45 \mathrm{RO}^{+} \mathrm{T}$ cells were obtained via positive selection using CD45RA or CD45RO Microbeads (Miltenyi). The purity of the naive CD8 (CD45RA ${ }^{+}$) and memory CD8(CD45RO ${ }^{+} \rrbracket$ was $\geq 95 \%$ as analyzed by flow cytometry. Isolated CD8 T cell subpopulations from both the patients and controls were frozen in $90 \% \mathrm{FCS} / 10 \% \mathrm{DMSO}$ in the vapor phase oliquid nitrogen until future use.

\section{DNA extraction and measurement of telomere length by quantitative real-time PCR}

After isolating naive and memory CD8 T cells, DNA was extracted using a QIAamp DNA Mini Kit (Qiagen) according to the manufacturers' instructions. The concentration and purity of the DNA was quantified using a Nanodrop Spectrophotometer (ThermoFisher Scientific, Waltham, Massachusetts, USA). The telomere length was determined with the widely used quantitative real-time PCR(qPCR) method[24]. The relative telomere length was measured as the ratio of standard DNA quantities for telomere template $(T)$ over single copy gene(SCG), human beta-globin. The telomere and SCG gene primers were as follows: (Tel-forward,5'-CGGTTTGTTTGGGTTTGGGTTTGGGTTTGGGTTTGGGTT-3'; Tel reverse, 5'GGCTTGCCTTACCCTTACCCTTACCCTTACCCTTACCCT-3') for telomere PCR and (SCG-forward, 5'GCTTCTGACACAACTGTGTTCACTAGC-3'; SCG-reverse, 5'-CACCAACTTCATCCACGTTC-ACC-3') for the 
SCG PCR. All samples were blindly and consecutively run in triplicate together with reference samples. The inter assay coefficient of variation was $<5 \%$. The formula to convert T/S ratio to base pairs was $3,274+2,413^{\star}(T / S)$. The conversion from $T / S$ ratio to bp was calculated based on a comparison of telomeric restriction fragment length from Southern blot analysis and T/S ratios using DNA samples from the human diploid fibroblast cell line IMR90 at different population doublings.

\section{Statistical analysis}

All continuous variables were expressed as the median with an interquartile range(IQR) or mean with standard deviation(SD), as appropriate. All groups were tested for the normal distribution with a Kolmogorov-Smirnov test. Differences between groups were analyzed by Student's t-test or the MannWhitney U test according to data distribution. All statistical analysis was carried out using Graphpad Prism version 5 (GraphPad, La Jolla, CA, USA) and SPSS 22.0 (College Station, TX, USA) software. In the graphs, the $P$ values are indicated as follows: $*<0.05 ; * \star<0.01 ; * \star \star<0.001$.

\section{Results}

\section{Characteristics of study population}

Characteristics of 26 ART-naive HIV-infected MSM and 20 age-matched HIV-seronegative MSM are reported in Table 1. The median age of the HIV-infected population was 28.5years (IQR 24.8-33.0 years), similar to the HIV-seronegative group (median 27.0 years (IQR 25.3-29.0 years) $(P=0.73)$. The CD4/CD8 ratio is a marker of immune dysfunction in the general population as well as in HIV-infected individuals[25]; therefore, we first evaluated CD4 and CD8 T cell counts and calculated the CD4/CD8 ratio. The median CD4 T cell counts were significantly reduced in the ART-naive MSM with a detectable viral load $(P<0.0001)$. Meanwhile, CD8 T cell counts were significantly elevated $(P=0.0058)$ and as expected, the CD4/CD8 ratio was significantly reduced accordingly $(P<0.0001)$ in ART-naive MSM compared with HCs.

Table 1

Characteristics of the study population

\begin{tabular}{|c|c|c|c|}
\hline & $\operatorname{HCs}(n=20)$ & ART-naive(n = 26) & $P$ \\
\hline Age, median (IQR) years & $27.0(25.3,29.0)$ & $28.5(24.8,33.0)$ & 0.73 \\
\hline CD4 count, median (IQR) cells/ul & $907(840,1024)$ & $290(183,441)$ & $<0.0001$ \\
\hline CD8 count, median (IQR) cells/ul & $545(493,681)$ & $846(531,1724)$ & 0.0058 \\
\hline CD4/CD8 ratios, median (IQR) & $1.70(1.53,1.80)$ & $0.30(0.16,0.56)$ & $<0.0001$ \\
\hline Viral load, median (IQR)copies/ml & na & $20625(4063,100643)$ & na \\
\hline
\end{tabular}




\section{Accelerated immune senescence of total CD8 T cells in ART-naive HIV-infected MSM}

Accelerated T cell senescence, phenotypically described as loss of the costimulatory molecule CD28 and acquisition of the exhaustion marker CD57, has been associated with HIV infection[5]. To assess the impact of HIV on immune senescence in HIV-infected MSM, we first analyzed the percentage of CD $28^{-} \mathrm{CD} 57^{+}$cells in total CD4 and CD8 T cells by multiparameter flow cytometry (Fig. 1 ). In our study cohort, the percentages of senescent cells $\left(\mathrm{CD} 28^{-} \mathrm{CD} 57^{+}\right)$in total CD4 T cell did not differ for both groups $(P=0.45)$. However, in total CD8 T cell, this percentage was more expanded in HIV-infected MSM than in controls $(P=0.03)$, thus supporting accelerated immune senescence in HIV-infected MSM.

\section{Alterations in the distribution of CD4 and CD8 T cell subsets in ART-naive HIV-infected MSM}

HIV infection is characterized by a disturbance in T cell homeostasis, which results in an alteration to T cell subsets. Next, we evaluated the impact of HIV on distribution of CD4 and CD8 T cell subsets in the participant groups.

Within CD4 T cell subsets, HIV-infected MSM and HCs did not significantly differ in percentages of TN, TCM, TEM and TemRA (Fig. 2). Notably, significant differences emerged among CD8 T cell subsets(Fig. 3). HIV-infected MSM showed lower percentage of naive cells than $\mathrm{HCs}(P=0.0046)$. Among memory cell subsets, a higher percentage of CD8 central memory cells was observed in HIV-infected $\operatorname{MSM}(P=0.0009)$. Similarly, both TEM and TemRA were more expanded in HIV-infected MSM than in controls $(P=0.020$ and $P=0.023$, respectively).

In summary, our cross-sectional data demonstrate HIV-infected MSM experimence a substantial reduction in the percentage of naive CD8 T cell subsets with a concomitant increase of memory CD8 T cell subsets. This may reflect altered CD8 T cell homeostasis and immunosenescence.

Increased senescence and activation phenotype in CD4 and CD8 T cell subsets in ART-naive HIV-infected MSM

We next analyzed alterations in the expression of replicative senescence and activation markers in distinct CD4 and CD8 T cell subsets.

A higher percentage of senescent cells $\left(\mathrm{CD} 28^{-} \mathrm{CD} 57^{+}\right)$in CD4 T cell subsets was observed in HIV-infected MSM compared to uninfected controls, with this difference being significant only for CD4 naive cells ( $P=$ 0.015) (Fig. 4). Notely, the phenotype of CD8 T cell subsets in HIV-infected MSM was also more senescent, with this increase statistically significant for all CD8 T cell subsets(Fig. 5), indicating a possible effect of HIV infection on the senescence of these cells.

Additionally, we analyzed the degree of activation in these CD4 and CD8 subsets and observed that an increase in HLA-DR expression in all CD4 and CD8 T cell subsets, with these alterations being significant 
(Fig. 4,5).

Collectively, our data demonstrate that HIV-infected MSM exhibit accelerated immune senescence which affects the CD8 $T$ cell subsets in particular.

\section{Shorter telomere length of naive and memory CD8 T cells in ART-naive HIV-infected MSM}

Telomeres are specialized structures comprised of tandem repeats of TTAGGG located at the end of chromosomes and are essential for chromosomal stability. An assessment of telomere length is used to evaluate cellular replication history or senescent status. Moreover, immune activation and microbial translocation are thought to drive telomere shortening in HIV infection[26]. Telomere shortening was consistently observed in the CD8 T cells, with only minimal or no telomere shortening in the CD4 T cells[27]. In order to investigate the impact of HIV infection on the telomere length of distinct T cell subsets, we further focused on determining the telomere length of naive and memory CD8 T cells isolated from HIV-infected MSM. The results are presented in Fig. 6. In addition to the fact that memory CD8 T cells were found to have a significantly shorter telomere length than naive CD8 T cells in HIV-infected MSM $(P<0.001)$, we observed that both naive and memory CD8 T cells in this population had significantly shorter telomere length than the same cell subsets from uninfected controls $(P<0.01)$, indicating more cell divisions in CD8 T cell subsets.

\section{Discussion}

This is the first study that shows an exhaustive analysis on immune senescence and premature aging of T cell subsets in ART-naive HIV-infected MSM. For this purpose, we measured not only the percentage, but also the degree of senescence and activation of these cells. Our findings provide evidence of accelerated immune senescence with biological aging in ART-naive HIV-infected MSM, which particularly affects CD8 T cell subsets .

The persistence of HIV induces activation of immune system cells, which undergo continuous expansion as a response to the antigen, eventually reaching the senescent stage, when they lose their functions[5]. As reported in many previous studies[21, 28, 29], our results show that HIV-infected MSM has a higher percentage of senescence marker in the total CD8 population compared to age-matched controls. In contrast to the study of Méndez-Lagares $\mathrm{G}$ et al.[30], we found no difference of senescence phenotype in the total CD4 population between two groups. This discordant result may be because of the different population characteristics of the two cohorts. Moreover, our results also demonstrate that presence of viral load in HIV-infected MSM favors the accumulation of CD8 T cells with memory and effector phenotypes and increases the degree of senescence in these CD8 T cells.

Although the CD4 T cell is the target of HIV infection, we found that CD8 T cell compartment was largely impaired in the HIV-infected MSM. Firstly, we analyzed the naive T population that is crucial to maintain the T cell pool. As expected, the results of this study showed lower percentage of naive CD8 T cells in HIVinfected MSM compared to uninfected controls. This may be partially explained by the capacity of the 
thymus to produce new T cells or thymic output, which is significantly decreased during the course of HIV infection[31-33]. Moreover, our results indicate that naive CD8 T cells in HIV-infected MSM are more activated and show a more replicative senescence phenotype. In presence of antigenic stimulus and absence of an adequate thymic output of naive cells, increased turnover of the CD8 population lead to the senescence of naive CD8 pool, which can be reflected by an increase in this replicative senescence phenotype. In conclusion, HIV-mediated impairment of thymic production could result in a deficiency in the replenishment of the naive CD8 pool in HIV-infected patients, thus accelerating immune senescence of naive CD8 T cells.

As already described in adults and older children[20,34,35], a skewed T-cell phenotype from naive toward effector memory induces accumulation of cells with a senescence phenotype. In this study, we observed a significantly higher percentage of CD8 memory T cell subsets with a more activated phenotype in HIVinfected MSM than uninfected controls. This profile may reflect the immune activation that drives cells into the state of terminal differentiation. Additionly, HIV-infected MSM show a more senescent phenotype of CD8 memory T cell subsets. These results indicate that as described in children, HIV infection induces the premature aging of the memory CD8 T-cell pool, eventually leading to the exhaustion and the depletion of these cells described in the progression of the disease[36].

On the other hand, telomeres are involved in cellular aging and immune senescence mechanisms. The shortening of telomere length is often used as an indicator of premature biological aging[37]. Our findings of shorter telomere length of naive and memory CD8 T cells in ART-naive HIV-infected MSM suggest that CD8 T cell subsets are chronically stimulated in these patients and that these cells have likely gone through a larger number of cell divisions. For HIV-infected subjects, cell division of naive and memory $T$ cells is required to the maintenance of $T$ cell compartments by homeostasis. This result is compatible with accelerated immune senescence of CD8 T cell subsets. Increased telomerase activity can compensate for the shortening of telomeres. However, the enzymatic activity of telomerase varies among different lymphocyte subsets[38,39]. Whether telomere shortening in naive and memory CD8 T cells is due to immune cell type-specific telomerase activity is still unclear, and it is interesting to clarify this issue in further studies.

In this study, we analyzed the immune senescence and biological aging of T cell subsets in ART-naive HIV-infected MSM. Unfortunately, our current cohort is too small to stratify these HIV-infected MSM based on laboratory parameters. Due to the design of our study and limited sample size, the data drawn decrease the power to provide statistically significant conclusions. Further studies are needed not only to confirm these data but also to ascertain the impact of HIV on the aging of HIV-infected MSM.

\section{Conclusions}

ART-naive HIV-infected MSM exhibit accelerated immune senescence with premature biological aging, particularly affects the CD8 T cell subsets. Compared with healthy controls, the shorter telomere length and higher percentage of senescent and activated cells in HIV-infected MSM with detectable HIV viremia 
highlight the strong impact of HIV on aging process of T cell subsets despite patient's young age at infection and support the importance of early control of HIV replication.

\section{Abbreviations}

MSM: Men who have sex with men; HIV: Human immunodeficiency virus; AIDS: Acquired immune deficiency syndrome; ART, antiretroviral therapy; PBMC, peripheral blood mononuclear cell; TN, naive T; TCM, central memory $\mathrm{T}$; TEM, effector memory $\mathrm{T}$; TemRA, terminally differentiated memory $\mathrm{T} ; \mathrm{HCs}$, healthy controls; IQR, interquartile range; SD, standard deviation.

\section{Declarations}

\section{Acknowledgements}

We thank the study participants and the staff that collected the blood samples at the Clinical and Research Center of Infectious Disease, Beijing Ditan Hospital.

\section{Authors ' contributions}

LL and LXW conceived and designed the study. LL, MCJ,YFT and SSJ carried out the study. LLand MCJ drafted the manuscript. WLH and TYX coordinated the research and revised the paper. LXW revised the final version of the manuscript. All authors approved the final manuscript.

\section{Funding}

This work was funded by National Science and Technology Major Project during the '13th Five-year' Plan Period (No.2017ZX10205502).

\section{Availability of data and materials}

The datasets used during the current study are available from the corresponding author on reasonable request.

\section{Ethics approval and consent to participate}

The protocol of this study was reviewed and approved by the Ethics Committee of the Beijing Ditan Hospital. Written informed consent was obtained from all participants.

\section{Consent for publication}

Not applicable.

\section{Conflict of interest}

The authors declare that there are no conflicts of interest. 


\section{References}

1. Bhatia R, Ryscavage $P$, Taiwo $B$. Accelerated aging and human immunodeficiency virus infection: Emerging challenges of growing older in the era of successful antiretroviral therapy. J Neurovirol. 2012;18:247-55.doi:10.1007/s13365-011-0073-y.

2. Deeks SG. Immune dysfunction, inflammation, and accelerated aging in patients on antiretroviral therapy. Top HIV Med. 2009;17:118-23 .

3. Capeau J. Premature aging and premature age-related comorbidities in HIV-Infected patients: facts and hypotheses. Clin Infect Dis. 2011;53:1127-9.doi:10.1093/cid/cir628.

4. Molina-Pinelo S, Vallejo A, Díaz L, Soriano-Sarabia N, Ferrando-Martínez S, Resino S, et al. Premature immunosenescence in HIV-infected patients on highly active antiretroviral therapy with low-level CD4 T cell repopulation. J Antimicrob Chemother. 2009;64:579-88.doi:10.1093/jac/dkp248.

5. Desai S, Landay A. Early Immune Senescence in HIV Disease. Curr HIV/AIDS Rep. 2010;7:410.doi:10.1007/s11904-009-0038-4.

6. Deeks SG. HIV infection, inflammation, immunosenescence, and aging. Annu Rev Med. 2011;62:14155.doi:10.1146/annurev-med-042909-093756.

7. Bischof J, Gärtner F, Zeiser K, Kunz R, Schreiner C, Hoffer E, et al. Immune Cells and Immunosenescence. Folia Biol (Praha). 2019;65:53-63 .

8. Appay V, Sauce D. Immune activation and inflammation in HIV-1 infection: causes and consequences. J Pathol. 2010;214:231-41.doi: 10.1002/path.2276.

9. Douek DC, Mcfarland RD, Keiser PH, Gage EA, Massey JM, Haynes BF, et al. Changes in thymic function with age and during the treatment of HIV infection. Nature. 1998;396:690-5. doi:10.1038/25374.

10. Kelley CF, Kitchen CMR, Hunt PW, Rodriguez B, Hecht FM, Kitahata M, et al. Incomplete peripheral CD4+ cell count restoration in HIV-infected patients receiving long-term antiretroviral treatment. Clin Infect Dis. 2009;48:787-94.doi:10.1086/597093.

11. Olovnikov AM. Telomeres, telomerase, and aging: origin of the theory. Exp Gerontol. 1996;31:443-8. doi:10.1016/0531-5565(96)00005-8.

12. Fumagalli M, Rossiello F, Clerici M, Barozzi S, Cittaro D, Kaplunov JM, et al. Telomeric DNA damage is irreparable and causes persistent DNA-damage-response activation. Nat Cell Biol. 2012;14:355-65. doi:10.1038/ncb2466.

13. Hewitt G, Jurk D, Marques FD, Correia-Melo C, Hardy T, Gackowska A, et al. Telomeres are favoured targets of a persistent DNA damage response in ageing and stress-induced senescence. Nat Commun. 2012;3:708. doi:10.1038/ncomms1708.

14. Armanios M, Blackburn EH. The telomere syndromes. Nat Rev Genet. 2012;13:693-704. doi:10.1038/nrg3246.

15. Pathai S, Lawn SD, Gilbert CE, McGuinness D, McGlynn L, Weiss HA, et al. Accelerated biological ageing in HIV-infected individuals in South Africa: a case-control study. AIDS. 2013;27:2375-84. 
doi:10.1097/QAD.0b013e328363bf7f.

16. Srinivasa S, Fitch KV, Petrow E, Burdo TH, Williams KC, Lo J, et al. Soluble CD163 is associated with shortened telomere length in HIV-infected patients. J Acquir Immune Defic Syndr. 2014;67:414-8. doi:10.1097/QAI.0000000000000329.

17. Zanet DL, Thorne A, Singer J, Maan EJ, Sattha B, Le Campion A, et al. Association between short leukocyte telomere length and HIV infection in a cohort study: No evidence of a relationship with antiretroviral therapy. Clin Infect Dis. 2014;58:1322-32. doi:10.1093/cid/ciu051.

18. Liu JC, Leung JM, Ngan DA, Nashta NF, Guillemi S, Harris M, et al. Absolute leukocyte telomere length in HIV-infected and uninfected individuals: evidence of accelerated cell senescence in HIV-associated chronic obstructive pulmonary disease. PLoS One. 2015;10:e0124426. doi:10.1371/journal.pone.0124426.

19. Gonzalez-Serna A, Ajaykumar A, Gadawski I, Muñoz-Fernández MA, Hayashi K, Harrigan PR, et al. Rapid Decrease in Peripheral Blood Mononucleated Cell Telomere Length After HIV Seroconversion, but Not HCV Seroconversion. J Acquir Immune Defic Syndr. 2017;76:e29-29e32. doi:10.1097/QAI.0000000000001446.

20. Díaz L, Méndez-Lagares G, Correa-Rocha R, Pacheco YM, Ferrando-Martínez S, Ruiz-Mateos E, et al. Detectable viral load aggravates immunosenescence features of CD8 T-cell subsets in vertically HIVinfected children. J Acquir Immune Defic Syndr. 2012;60:447-54.

doi:10.1097/QAI.0b013e318259254f.

21. Gianesin K, Noguera-Julian A, Zanchetta M, Del Bianco P, Petrara MR, Freguja R, et al. Premature aging and immune senescence in HIV-infected children. AIDS. 2016;30:1363-73. doi:10.1097/QAD.0000000000001093.

22. Duffau P, Wittkop L, Lazaro E, le Marec F, Cognet C, Blanco P, et al. Association of immune-activation and senescence markers with non-AIDS-defining comorbidities in HIV-suppressed patients. AIDS. 2015;29:2099-108. doi:10.1097/QAD.0000000000000807.

23. De MM, Rossi ME, Azzari C, Gelli MG, Galli L, Vierucci A. Different meaning of CD38 molecule expression on CD4+ and CD8+ cells of children perinatally infected with human immunodeficiency virus type 1 infection surviving longer than five years. Pediatr Res. 1998;43:752-8 . doi: 10.1203/00006450-199806000-00007.

24. Axelrad MD, Budagov T, Atzmon G. Telomere length and telomerase activity; a Yin and Yang of cell senescence. J Vis Exp. 2013:e50246. doi:10.3791/50246.

25. Lu W, Mehraj V, Vyboh K, Cao W, Li T, Routy JP. CD4:CD8 ratio as a frontier marker for clinical outcome, immune dysfunction and viral reservoir size in virologically suppressed HIV-positive patients. J Int AIDS Soc. 2015;18:20052. doi:10.7448/IAS.18.1.20052.

26. Deeks SG, Verdin E, Mccune JM. Immunosenescence and HIV. Curr Opin Immunol. 2012;24:501-6 .doi: 10.1016/j.coi.2012.05.004.

27. Wolthers KC, Bea G, Wisman A, Otto SA, de Roda Husman AM, Schaft N, et al. T cell telomere length in HIV-1 infection: no evidence for increased CD4+ T cell turnover. Science. 1996;274:1543-7. 
doi:10.1126/science.274.5292.1543.

28. Álvarez S, Brañas F, Sánchez-Conde M, Moreno S, López-Bernaldo de Quirós JC, Muñoz-Fernández MÁ. Frailty, markers of immune activation and oxidative stress in HIV infected elderly. PLoS One. 2020;15:e0230339. doi:10.1371/journal.pone.0230339.

29. Le Priol Y, Puthier D, Lécureuil C, Combadière C, Debré P, Nguyen C, et al. High cytotoxic and specific migratory potencies of senescent CD8+ CD57+ cells in HIV-infected and uninfected individuals. J Immunol. 2006;177:5145-54. doi:10.4049/jimmunol.177.8.5145.

30. Méndez-Lagares G, Díaz L, Correa-Rocha R, León Leal JA, Ferrando-Martínez S, Ruiz-Mateos E, et al. Specific patterns of CD4-associated immunosenescence in vertically HIV-infected subjects. Clin Microbiol Infect. 2013;19:558-65. doi:10.1111/j.1469-0691.2012.03934.x.

31. Dion ML, Poulin JF, Bordi R, Sylvestre M, Corsini R, Kettaf N, et al. HIV infection rapidly induces and maintains a substantial suppression of thymocyte proliferation. Immunity. 2004;21:757-68. doi:10.1016/j.immuni.2004.10.013.

32. Fabre-Mersseman V, Dutrieux J, Louise A, Rozlan S, Lamine A, Parker R, et al. CD4\ recent thymic emigrants are infected by HIV in vivo, implication for pathogenesis. AIDS. 2011;25:1153-62. doi:10.1097/QAD.0b013e3283471e89.

33. Lelièvre JD, Melica G, Itti E, Lacabaratz C, Rozlan S, Wiedemann A, et al. Initiation of C-ART in HIV-1 infected patients is associated with a decrease of the metabolic activity of the thymus evaluated using FDG-PET/computed tomography. J Acquir Immune Defic Syndr. 2012;61:56-63 .doi: 10.1097/QAl.0b013e3182615b62.

34. Brenchley JM, Karandikar NJ, Betts MR, Ambrozak DR, Hill BJ, Crotty LE, et al. Expression of CD57 defines replicative senescence and antigen-induced apoptotic death of CD8+ T cells. Blood. 2003;101:2711-20 .doi: 10.1182/blood-2002-07-2103.

35. Montesano C, Anselmi A, Palma P, Bernardi S, Cicconi R, Mattei M, et al. HIV replication leads to skewed maturation of CD8-positive T-cell responses in infected children. New Microbiol. 2010;33:3039 .

36. Yamamoto T, Price DA, Casazza JP, Ferrari G, Nason M, Chattopadhyay PK, et al. Surface expression patterns of negative regulatory molecules identify determinants of virus-specific CD8+ T-cell exhaustion in HIV infection. Blood. 2011;117:4805-15. doi:10.1182/blood-2010-11-317297.

37. Appay V, Sauce D. Assessing immune aging in HIV-infected patients. Virulence. 2017;8:529-38. doi:10.1080/21505594.2016.1195536.

38. Ouyang Q, Baerlocher G, Vulto I, Lansdorp PM. Telomere length in human natural killer cell subsets. Ann N Y Acad Sci. 2007;1106:240-52. doi:10.1196/annals.1392.001.

39. Weng NP, Granger $L$, Hodes RJ. Telomere lengthening and telomerase activation during human $B$ cell differentiation. Proc Natl Acad Sci USA. 1997;94(20):10827-32.doi: 10.1073/pnas.94.20.10827.

\section{Figures}


(a)

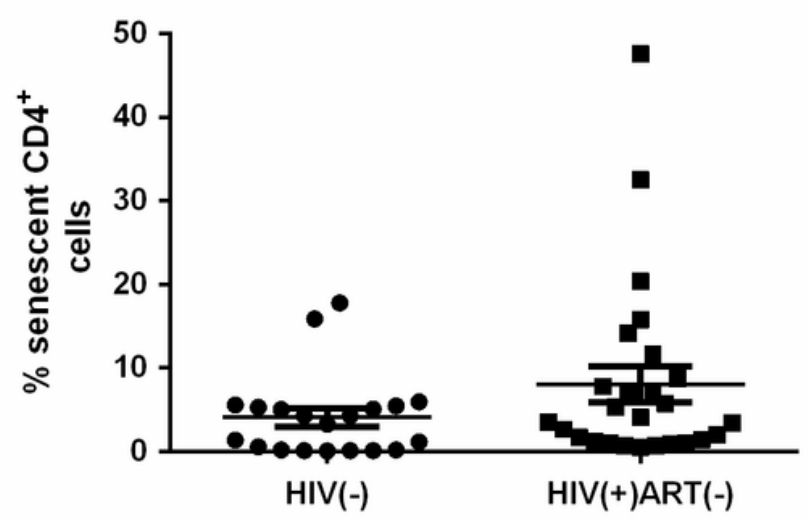

(b)

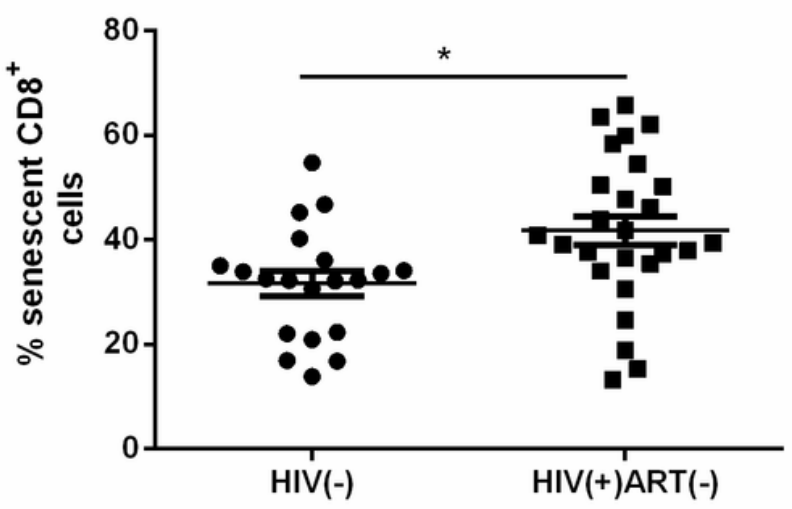

Figure 1

To assess the impact of HIV on immune senescence in HIV-infected MSM, we first analyzed the percentage of CD28-CD 57+ cells in total CD4 and CD8 T cells by multiparameter flow cytometry

(a)

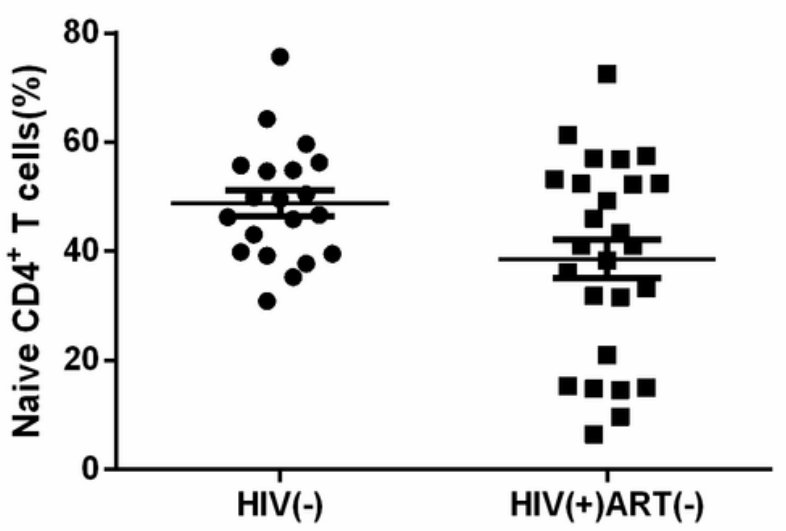

(c)

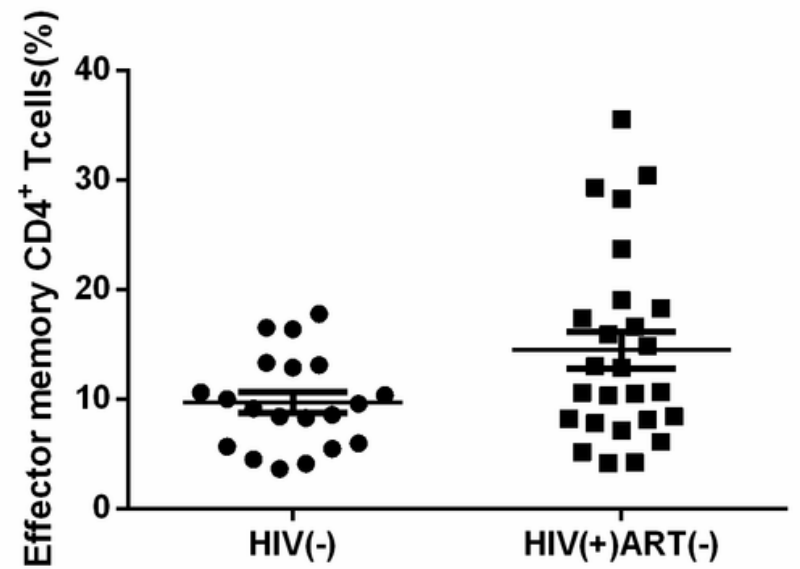

(b)

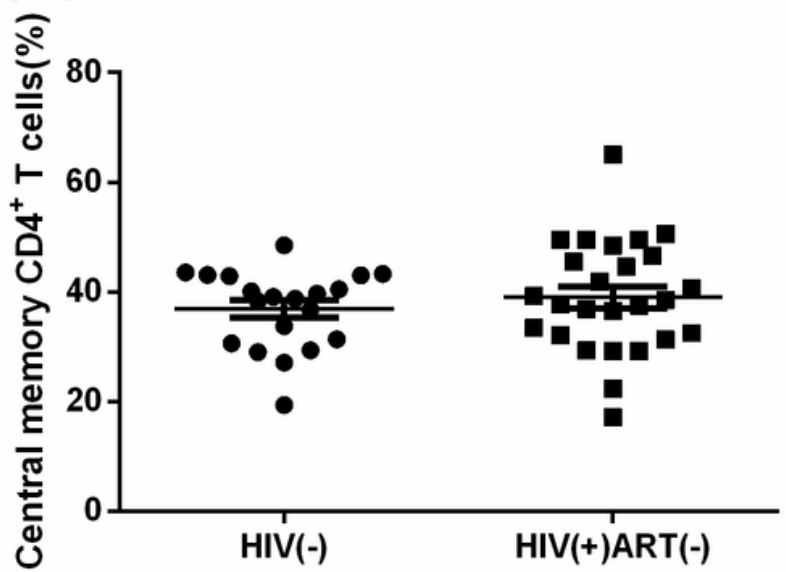

(d)

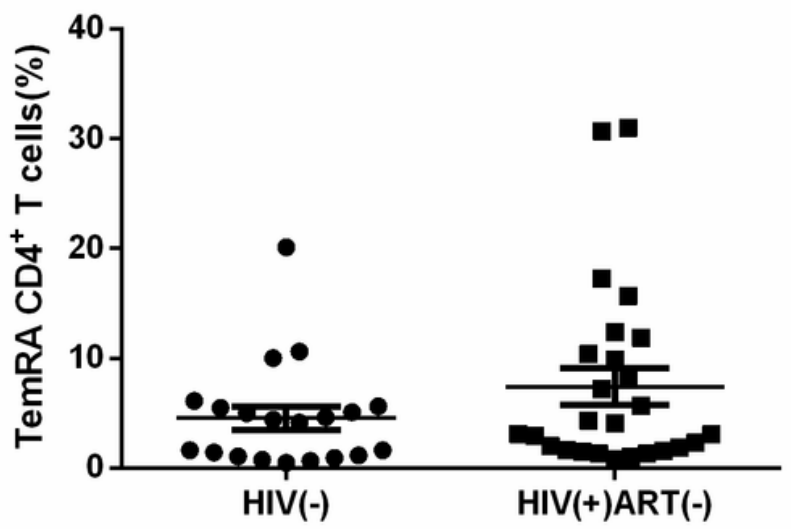

Figure 2 
HIV-infected MSM and HCs did not significantly differ in percentages of TN, TCM, TEM and TemRA

(a)

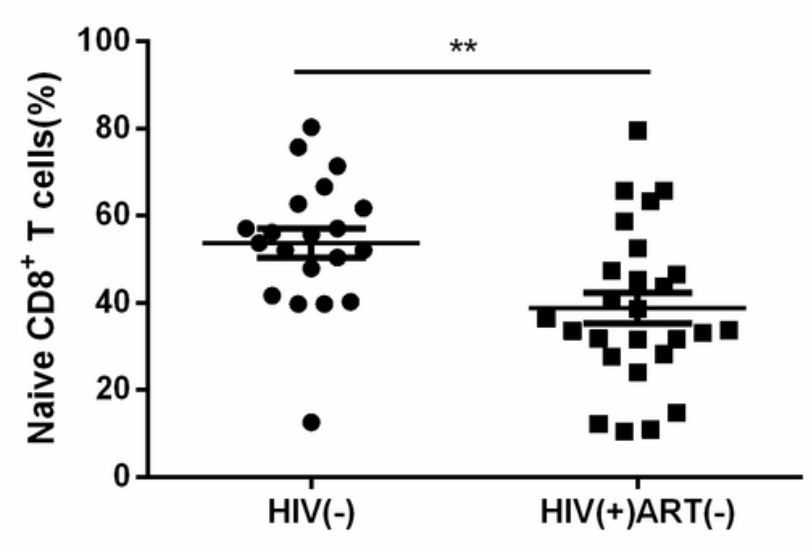

(c)

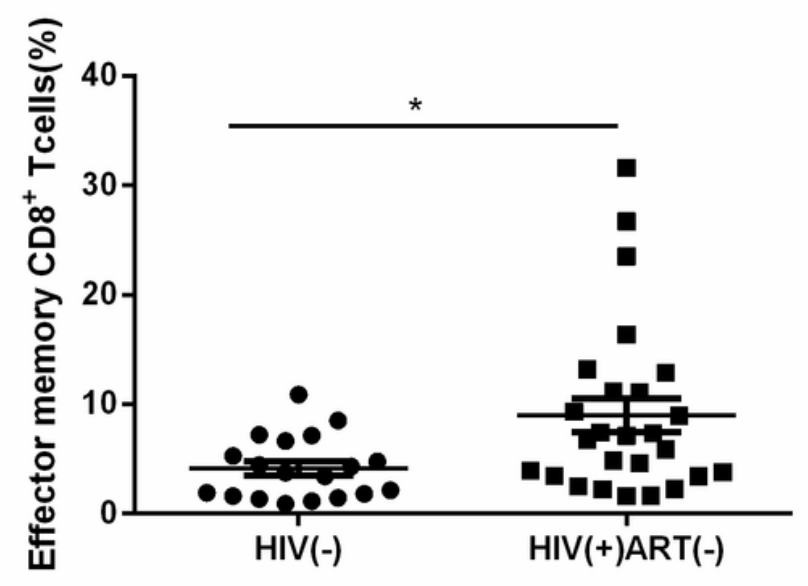

(b)

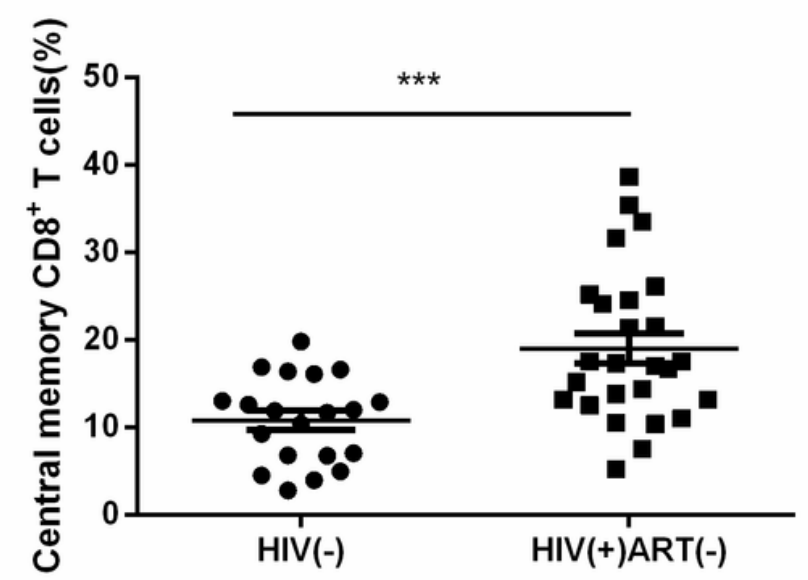

(d)

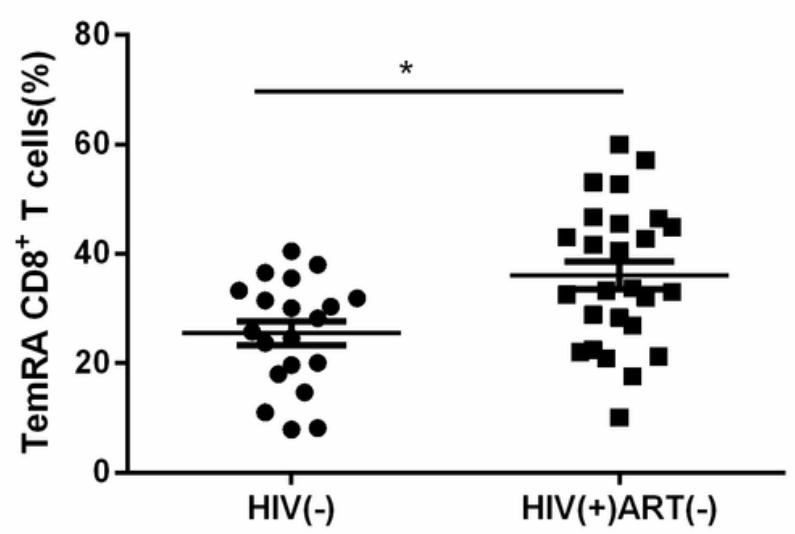

Figure 3

significant differences emerged among CD8 T cell subsets 
(a)

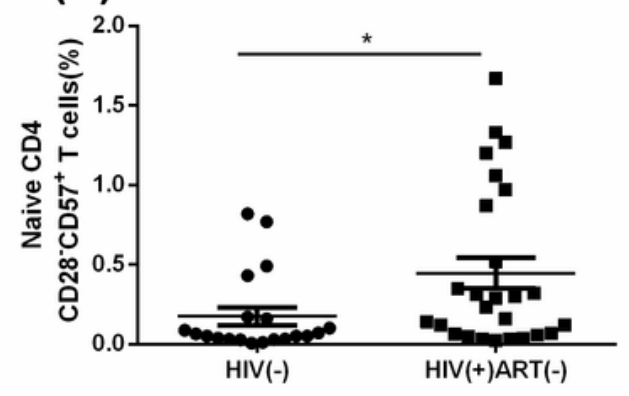

(c)

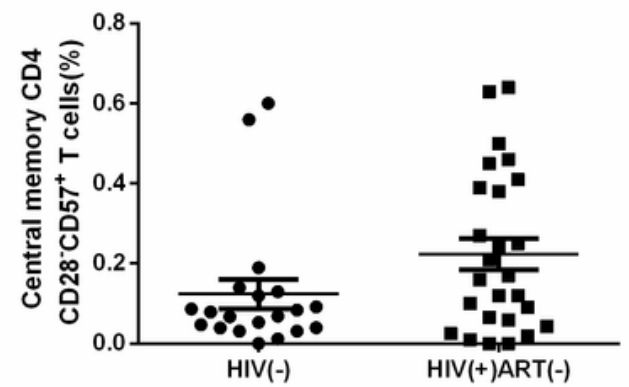

(e)

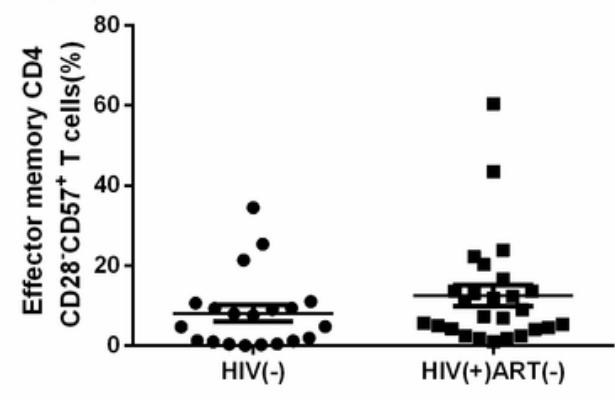

(g)

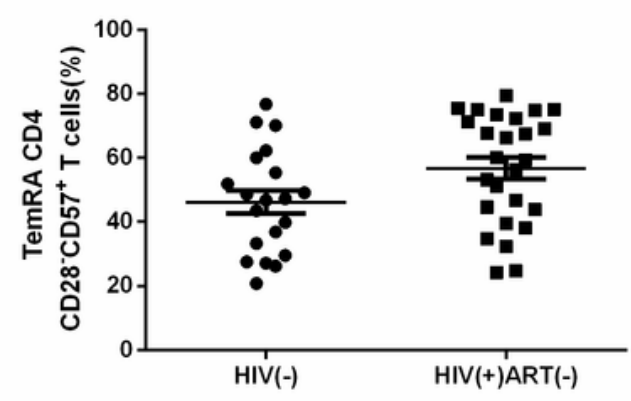

(b)

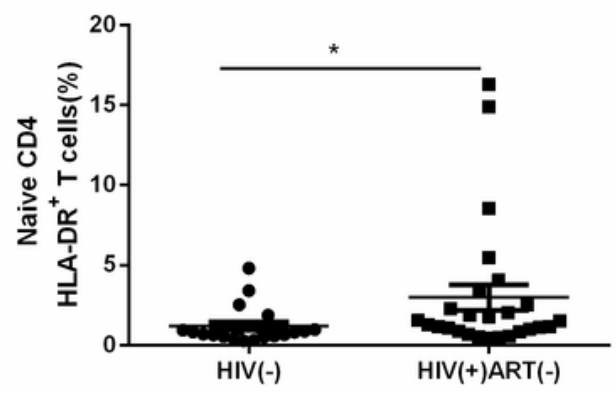

(d)

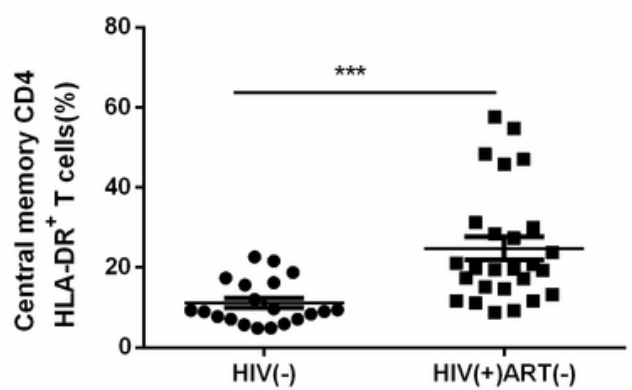

(f)

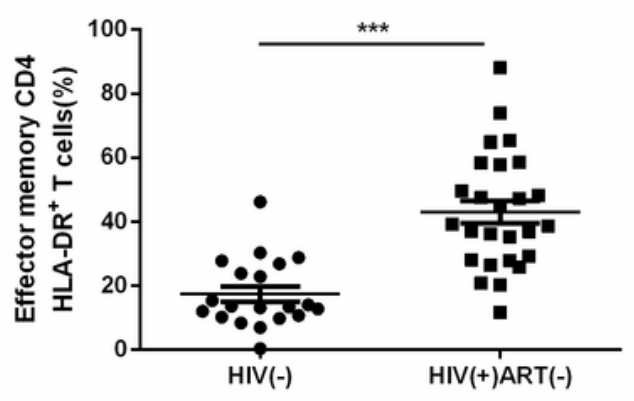

(h)

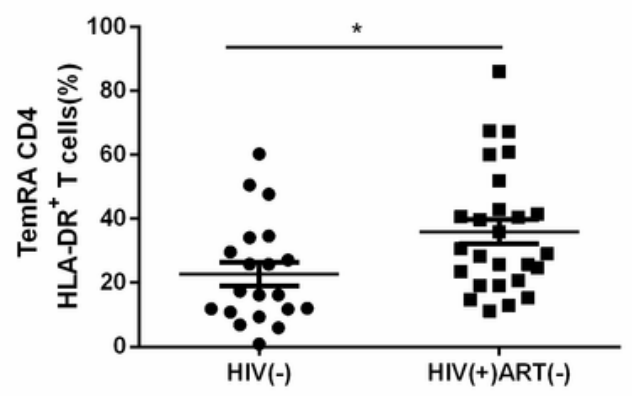

Figure 4

A higher percentage of senescent cells (CD28-CD57+) in CD4 T cell subsets was observed in HIV-infected MSM compared to uninfected controls, with this difference being significant only for CD4 naive cells $(\mathrm{P}=0.015)$ 
(a)

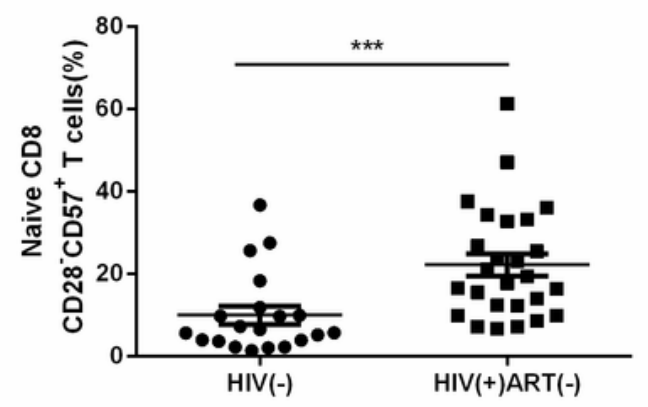

(c)

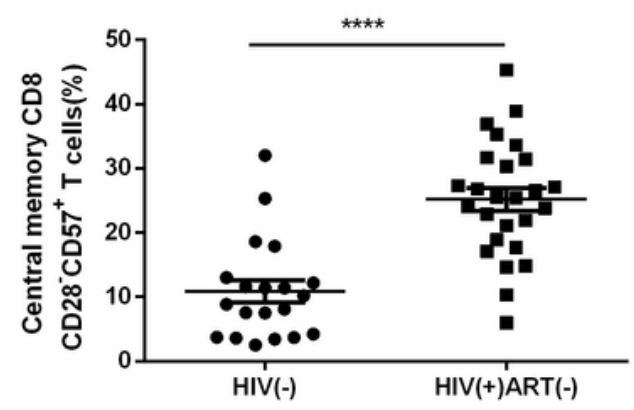

(e)

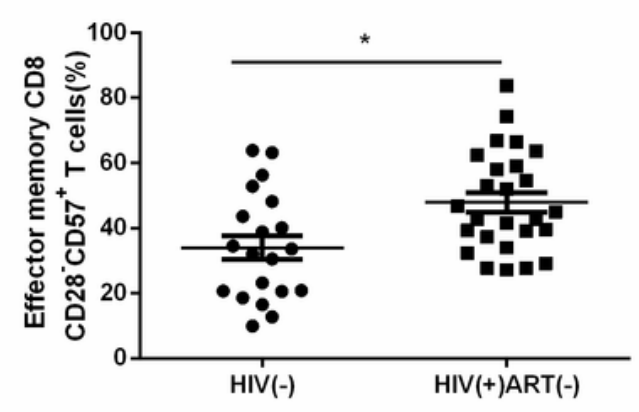

(g)

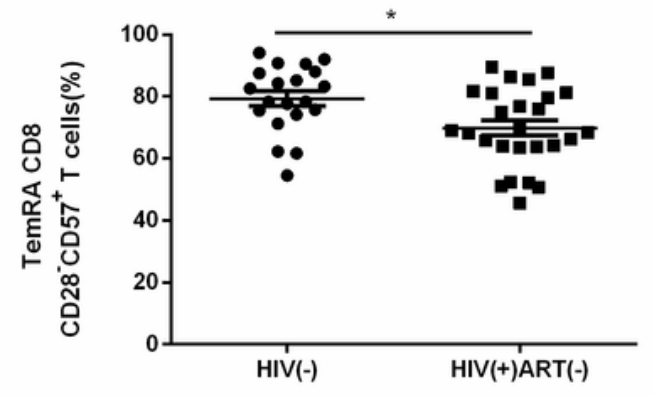

(b)

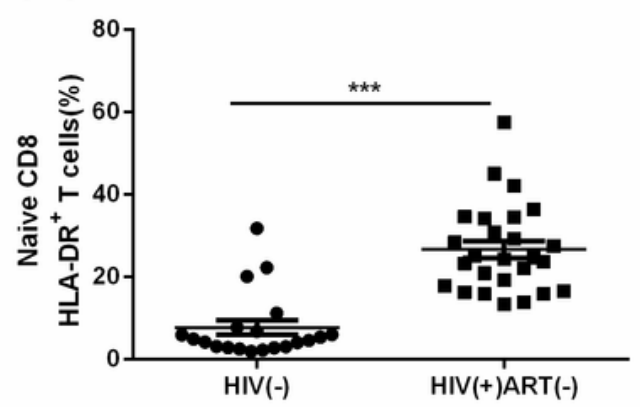

(d)

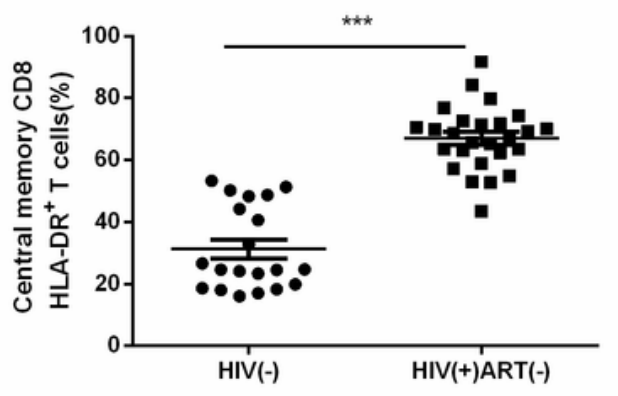

(f)

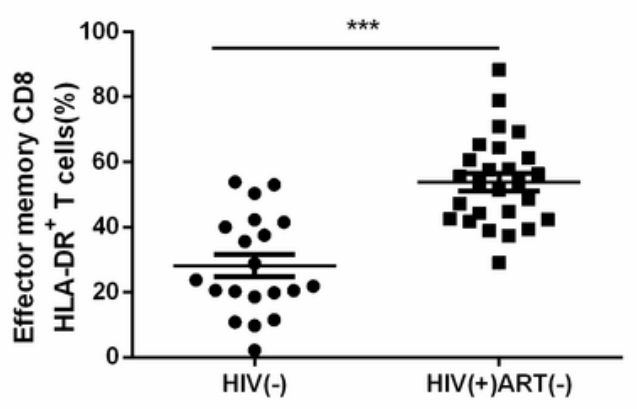

(h)

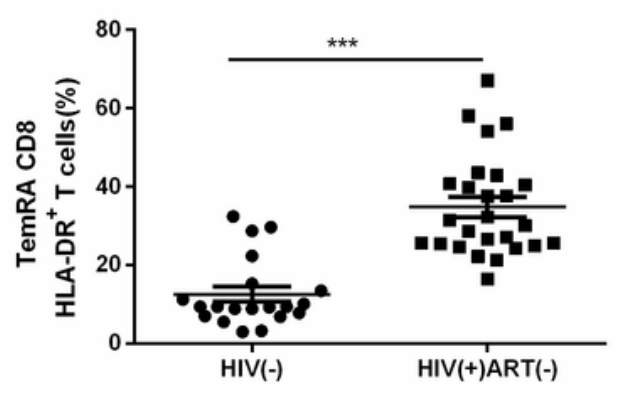

Figure 5

the phenotype of CD8 T cell subsets in HIV-infected MSM was also more senescent, with this increase statistically significant for all CD8 T cell subsets 


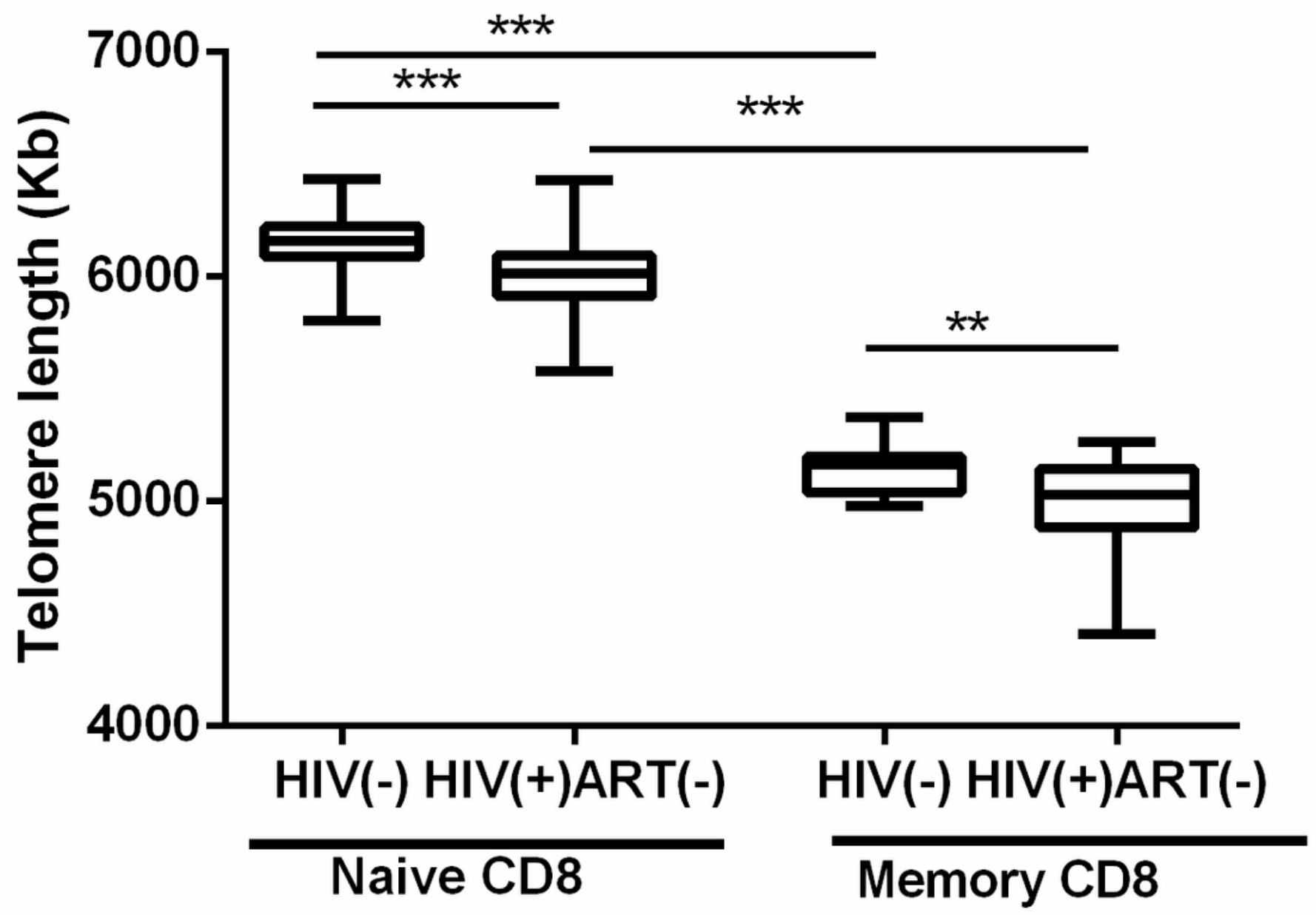

Figure 6

Shorter telomere length of naive and memory CD8 T cells in ART-naive HIV-infected MSM 